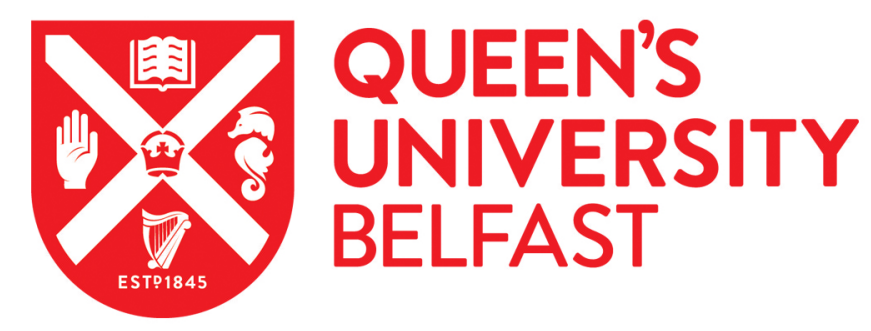

\title{
Development of a conceptual framework to improve sexual wellbeing communication in routine prostate cancer care
}

\author{
McCaughan, E., Parahoo, K., Flannagan, C., Maguire, R., Connaghan, J., Steele, M., Thompson, S., Jain, S., \\ Kirby, M., Brady, N., \& O'Connor, S. R. (2020). Development of a conceptual framework to improve sexual \\ wellbeing communication in routine prostate cancer care. Patient Education and Counseling. \\ https://doi.org/10.1016/j.pec.2020.01.014
}

Published in:

Patient Education and Counseling

Document Version:

Peer reviewed version

Queen's University Belfast - Research Portal:

Link to publication record in Queen's University Belfast Research Portal

\section{Publisher rights}

Copyright 2020 Elsevier Ltd.

This manuscript is distributed under a Creative Commons Attribution-NonCommercial-NoDerivs License

(https://creativecommons.org/licenses/by-nc-nd/4.0/), which permits distribution and reproduction for non-commercial purposes, provided the author and source are cited.

\section{General rights}

Copyright for the publications made accessible via the Queen's University Belfast Research Portal is retained by the author(s) and / or other copyright owners and it is a condition of accessing these publications that users recognise and abide by the legal requirements associated with these rights.

Take down policy

The Research Portal is Queen's institutional repository that provides access to Queen's research output. Every effort has been made to ensure that content in the Research Portal does not infringe any person's rights, or applicable UK laws. If you discover content in the Research Portal that you believe breaches copyright or violates any law, please contact openaccess@qub.ac.uk. 


\section{Development of a conceptual framework to improve sexual wellbeing communication in routine prostate cancer care}

Eilís McCaughan*1., Kader Parahoo ${ }^{1}$., Carrie Flannagan¹., Roma Maguire ${ }^{2}$., John Connaghan ${ }^{2}$., Mary Steele ${ }^{3}$., Samantha Thompson ${ }^{4}$., Suneil Jain ${ }^{5,6}$., Mike Kirby ${ }^{7}$., Nuala Brady8., Seán R O'Connor ${ }^{1,9}$.

* Corresponding author

1. Institute of Nursing and Health Research, Ulster University, Jordanstown, Northern Ireland, UK.

2. Department of Computer and Information Sciences, University of Strathclyde, Glasgow, UK.

3. Centre for Clinical and Community Applications of Health Psychology, Psychology, Faculty of Social and Human Sciences, University of Southampton, Southampton, UK.

4. Urology Department, Belfast City Hospital, Belfast, Northern Ireland, UK.

5. Centre for Cancer Research and Cell Biology, Queen's University Belfast, Belfast, Northern Ireland, UK.

6. Clinical Oncology, Northern Ireland Cancer Centre, Belfast City Hospital, Belfast, Northern Ireland, UK.

7. Faculty of Health and Human Sciences, University of Hertfordshire and The Prostate Centre, London, UK.

8. Northern Health and Social Care Trust, Northern Ireland, UK.

9. Centre for Public Health, Queen's University Belfast, Belfast, Northern Ireland, UK.

Corresponding author at:

Eilís McCaughan

Professor in Cancer Care

Institute of Nursing and Health Research

Ulster University

Coleraine, Co Derry

$\mathrm{N}$ Ireland 
BT52 1SA

Telephone: 02870124091

Email: em.mccaughan@ulster.ac.uk 


\section{Abstract}

\section{Objective:}

To systematically develop a framework to improve sexual wellbeing communication in routine prostate cancer care.

\section{Methods:}

The Theoretical Domains Framework was used to guide a multi-phase process used to identify components of the framework based on evidence reviews, semi-structured interviews and stakeholder workshops. 'Think-aloud' testing was used to explore usability, potential barriers and other factors relevant to implementation.

\section{Results:}

A conceptual communication framework consisting of 'Engagement' (E), 'Assessment' (A), information and 'Support' (S) and 'Sign-posting' (Si) sections was developed. The framework emphasises routine engagement to normalise sexual concerns, brief, nonsensitive assessment, personalised advice based on treatment and relationship status, and a mechanism for referral to additional support or self-management resources in the form of a patient and partner handout. Usability testing identified strategies to promote implementation.

\section{Conclusions:}

The proposed framework is appropriate for use in routine practice and appears to be acceptable to patients, partners and healthcare professionals. Its use may help address gaps in sexual wellbeing support for men and partners living with prostate cancer. Further work will be conducted evaluating an online engagement tool, modelled on the framework.

\section{Practice Implications:}

The EASSi framework can facilitate and structure sexual wellbeing conversations and ensure fundamental but individualised support is provided routinely in prostate cancer care.

Keywords: Communication; Sexual Wellbeing; Quality of life; Prostate cancer 


\section{Introduction}

Prostate cancer is the most common cancer among men [1,2]. As a result of increasing survival rates, long-term side-effects associated with different treatment approaches are common [3]. In a recent, large scale survey, proximately $80 \%$ of men reported poor sexual function post-treatment [4]. Changes to sexual function are a major issue in the post-treatment phase and can result in high levels of anxiety, depression, relational dis-satisfaction and reduced overall quality of life [5-7].

Treatment guidelines [8,9] endorse delivery of psychosexual care for prostate cancer patients with recommendations made for the minimal level of support that should be provided. This includes provision of individualised information tailored to need, clear advice about potential long-term side-effects of treatment, and ensuring ongoing access to specialist care including erectile dysfunction clinics. Despite these recommendations, information varies greatly and is not provided routinely across services [4], with patients and partners frequently reporting that they do not receive adequate support to manage these concerns [10-12].

Initiating discussions around sexual concerns in clinical practice can be challenging. A number of potential barriers have been identified including structural factors such as limited contact time [13] or non-availability of onward referral services. Process factors influenced by inter-personal communication may also limit discussions further. For example, healthcare professionals often regard patients' sexual lives as being too personal to ask about and they may actively avoid the issue [14-16]. They also report that management of sexual issues is not within their professional role, and that they feel unequipped to deal with sexual issues, highlighting a lack of resources to offer patients if they do identify a problem [17]. Men themselves can feel uncertain about discussing concerns with healthcare professionals and may not be fully aware of the potential side-effects of treatment. Broader social influences and attitudes to sex and sexuality, including embarrassment, not being comfortable with the topic or not wishing to cause offence can also be barriers. However, given their frequency and substantial impact [4], sexual concerns should be discussed routinely with patients, and healthcare professionals are ideally placed to provide this support. 
For healthcare professionals to address sexual health and wellbeing needs of men living with prostate cancer, they require knowledge and skills to effectively engage with patients and assess needs to provide appropriate evidence-based management [18]. One approach which could support healthcare professional communication is use of written prompts or structured questions to guide conversations. For example, sexual dysfunction screening checklists could be used to identify needs [19]. Evidence-based frameworks or tools can also provide a mechanism to provide appropriate advice and information [20,21]. Such approaches may enhance patient-provider communication, particularly around complex or sensitive issues by ensuring a more standardised provision of information. For example, use of decision support tools in a variety of clinical settings have been found to increase knowledge and reduce decisional conflict [22]. However, there is currently limited evidence exploring how communication can be enhanced and how conversations around sexual wellbeing can be supported in routine practice. There is a clear need for approaches that are available at all phases of care and which provide all healthcare professionals with the skills and capacity to routinely deliver sexual care and support. It is important that any framework used to guide practice is clear, user-friendly and is able to be integrated into practice with limited training.

\subsection{Overview of existing sexual care models}

A number of sexual care models have previously been proposed and tested in clinical settings. These include the PLISSET [23], EX-PLISSIT [24], and BETTER [25] approaches. The EX-PLISSIT model [24], and its precedent, the PLISSIT model [23], have been the most widely examined. The PLISSIT model involves: asking for or giving patients 'Permission' to discuss sexual wellbeing, giving 'Limited Information', about sexual side-effects of treatment, making 'Specific Suggestions' based a full evaluation of current problems, and providing or referring to more 'Intensive Therapy' including psychosexual therapy. It is aimed at providing increasing levels of engagement or intensity. The BETTER model [25] is aimed at supporting health professionals to incorporate sexual wellbeing assessment in cancer care settings. The model includes: 'Bringing-up' the topic of sexual concerns, 'Explaining', or normalising the topic by framing it as an important quality-of-life issue, 'Telling' the patient about resources for addressing any sexual concerns, consideration of 'Timing', by ensuring any discussion accounts for the patient's readiness to talk about sexual issues, 
providing 'Education' including information on side-effects of treatment and lastly, 'Recording' or documenting the assessment, treatment and outcome, including followup plans, in patients' medical records. The above models provide differing approaches to sexual care and support. The PLISSIT model involves a counselling-based, stepped approach. For example, using a non-judgemental listening approach to determine patient's information needs [26]. The BETTER model uses behavioural and personbased methods including use of reminders or prompts, problem solving techniques and planning notes recorded in patients' clinical records [25].

\subsection{Rationale for a conceptual framework to guide brief sexual wellbeing conversations}

The need for a brief approach to ensure sexual wellbeing is routinely discussed was identified based on previous work by members of the project team [26-29], and through discussions with cancer care professionals and patient support groups. This process identified that the proposed framework should promote active engagement with all patients, include an assessment to provide essential information on potential sideeffects, and refer to appropriate ongoing support, including self-management approaches. Following a rapid evidence review and initial qualitative work with patients and healthcare professionals, a co-development approach was used to devise an initial outline for an engagement and support framework intended to facilitate routine, sexual wellbeing discussions in the clinical setting.

While existing care models such as PLISSIT and BETTER provide well developed and structured approaches, they have limited active behaviour change components to support effective implementation into practice. In addition, both models include aspects which may present limitations to ensuring brief sexual care discussions take place routinely. This includes that healthcare professionals are asked to seek explicit permission or approval to talk about sexual concerns at any point where it might be discussed and also consider timing by ensuring patients are ready to discuss sexual concerns.

A further concern is that these approaches may provide an 'opt-out' leading to healthcare professionals not initiating discussions based on a perception that the patient is not ready or does not wish to discuss sexual issues. Previous evidence 
indicates that men with prostate cancer and their partners do want healthcare professionals to discuss sexual issues and side-effects of treatment [30]. Despite this, sexual wellbeing discussions are often infrequent and limited in scope, with partners often not involved in any conversations that do take place [31]. Furthermore, healthcare professionals using judgement to determine when it is appropriate to discuss sexual concerns with patients can be problematic. The sensitivity of the topic and structural factors in current care systems, including that patients may not see the same healthcare professional at each appointment can mean that even essential advice and support on the impact of treatment is not discussed with a patient at any stage in their care. This highlights a need for a framework which supports healthcare professionals to routinely initiate sexual wellbeing discussion and, addresses barriers to such discussions taking place. In addition, the framework should normalise sexual issues in prostate cancer care, include partners and address expectations around recovery by introducing the concept of a 'new sexual health normal'. A framework that specifies and acknowledges the impact of treatment on sexual wellbeing of both men and their partners as well as providing appropriate support has potential to improve healthcare professional communication and patient-important outcomes.

\subsection{Aim}

The aim of this paper is to describe the systematic development of a conceptual framework designed to facilitate routine conversations about sexual wellbeing in prostate cancer care. Detail is provided on the rationale, evidence-base and theoretical underpinning of the framework. Iterative development, based on the views of patients, partners and healthcare professionals explored using qualitative methods is also described.

\section{Methods}

The Theoretical Domains Framework (TDF) [32] was used as a guide to identify key components of the proposed conceptual framework and explore barriers and factors relevant to implementation. The TDF includes 14 domains influencing clinical behaviour including knowledge, skills, professional role and identity, beliefs about capabilities, decision processes, environmental context and social influences.

\subsection{Ethics}


Ethical approval for the study was provided via the Office for Research Ethics Committees Northern Ireland (ORECNI) (Reference number: 17/NI/014). All participants gave written, informed consent before participation.

\subsection{Procedures}

Systematic evidence reviews, qualitative interview methods and stakeholder workshops were used to inform development of the proposed framework (see table 1 for a summary of qualitative methods used). The process consisted of four distinct phases. [i] Identifying changes needed to reduce the evidence-practice gap. [ii] Identifying barriers, facilitators and theories likely to explain necessary behaviour changes. [iii] Identifying appropriate, feasible and acceptable framework components, including modes of delivery, as well as behaviour change components likely to overcome barriers and assist facilitators. [iv] Identifying proposed mechanisms of change and selection of appropriate outcomes to measure change and evaluate the framework.

\subsection{Phase $i$. Identifying changes needed to reduce the evidence-practice gap}

The initial step in development involved a scoping review to explore requirements and expected components for each section of the framework. This was carried out to identify existing interventions and patient and healthcare professional perceived needs around sexual wellbeing communication in cancer care. Qualitative evidence was also gathered from individual, semi-structured interviews with key stakeholders, and findings from an interactive stakeholder workshop including men, partners, policy makers and healthcare professionals were used to identify requirements and expected components of the proposed framework (See table 2). As well as identifying the requirements of the framework, interviews with healthcare professionals and patients were used to assess views on current practice. All interviews were audio recorded, transcribed and analysed using deductive content analysis methods.

\subsection{Phase ii. Identifying barriers and facilitators, and theories likely to explain necessary behaviour changes}

To provide an overview of the theoretical assumptions underpinning the proposed framework, a mixed-method systematic review was conducted to identify the barriers to communication around sexual wellbeing in clinical practice [33]. Barriers and 
facilitators to sexual wellbeing discussions were explored further by conducting an additional series of interviews with men and partners, and group discussions with healthcare professionals.

\subsection{Phase iii. Identifying framework components likely to overcome barriers and assist facilitators}

Outline structure and content for the framework was reviewed by members of an expert group who were asked to comment on appropriateness of language, tone, quality and accuracy of information, as well as necessity of the content and mode of delivery. In addition, written content, including language and tone, were explored through individual semi-structured interviews with men, partners and healthcare professionals (see supplementary file 1 for interview topic guide). The framework was then tested by healthcare professionals, as part of a 'think- aloud' process facilitated by the lead author (EMcC). This preliminary framework was also tested with patients who were undergoing treatment (using a simulated conversation conducted outside of their routine care appointments). These discussions were observed, and perspectives were sought on framework content, and utility. Selection of behaviour change components was based on relevance, and perceived feasibility of implementing the framework in practice.

\subsection{Phase iv. Identifying proposed mechanisms of change and selection of appropriate outcome measures}

Proposed mechanisms by which use of the framework could lead to changes in practice were identified and used to select key outcome measures for evaluating the effectiveness of the framework. These outcomes included the extent to which use of the framework would improve healthcare professional beliefs and self-efficacy as well as patient satisfaction with use of the framework, and with the level of sexual wellbeing support provided (See figure 2).

\section{Results}

\subsection{Findings from evidence reviews}

The scoping review identified 21 studies reporting primarily on patient perceived needs and experiences of sexual wellbeing communication. Seven studies explored 
interventions designed to improve communication and support. Analysis of these interventions provided a summary of the different components and strategies used. The majority were not based on any underpinning theory or model and most were relatively long or intensive, including a number of separate sessions delivered over time. Most were also focused primarily on physical consequences and side-effects of treatment and did not account for different information needs. Patient perceived information and support needs included written information on different side-effects of treatment, as well as information for partners.

Data from the additional mixed-methods review which explored barriers to communication around sexual wellbeing in clinical practice were synthesised using a meta-ethnographic approach [33]. Fifty-seven, second-order concepts were extracted from 30 included studies. These were used to develop a conceptual framework based on five third order themes covering attitudes to sex and sexual wellbeing, patient factors, organizational factors, strategies to overcome barriers, and training needs. The review found healthcare professionals acknowledged the importance of discussing and providing support for sexual wellbeing needs, but recognised it is not routinely provided. Patient specific factors and organizational issues such as lack of time were frequently identified as barriers, however, intra-personal and social perceptions around sexual issues, including fear of embarrassment and assumptions made about age and sexuality appeared to have the strongest influence on healthcare professional perspectives. The review highlighted a need for brief educational and support tools to promote effective conversations with patients.

\subsection{Findings from qualitative interviews}

Data from the 76 participants including patients, partners and healthcare professionals involved in the qualitative evaluation (see table 1) identified that healthcare professionals and patients felt discussions around sexual wellbeing were limited and variable in content. Furthermore, it was acknowledged that discussions that did occur tended to have a biomedical focus centred on medication for erectile dysfunction and included limited support or onward referrals to address any other issues that did emerge. The experiences of patients and healthcare professionals also tended to differ, with healthcare professional typically being more satisfied with the content of sexual wellbeing discussions and the support offered. However, they did report a lack 
of resources to offer and did not use any framework or model to help guide sexual wellbeing conversations. Patients often reported not being aware of sex being discussed, particularly early after diagnosis. Discomfort with the topic of sexual wellbeing was perceived by some healthcare professionals to be a significant factor limiting the ability to assess and address the needs of men with prostate cancer, and their partners.

\subsection{Underpinning theories and behaviour components of the conceptual framework}

Appropriate theories and models have a critical role in identifying factors contributing to behavioural interventions. Social cognition theory is focused on an individual's motivation and actions based on situational outcomes, action outcomes and perceived self-efficacy [34-36]. The theory of reasoned action [37-39] was also seen as critical to the framework. In this context, this theory integrates both behavioural and normative beliefs and suggests that the most significant influencing factor is intention, which is determined by attitudes towards discussing sexual concerns in practice. This factor is strongly affected by personal beliefs around sex, as evidenced in the mixed-methods review conducted as part of the exploratory phase in this development process [33]. Intention is determined by favourable or unfavourable attitudes towards discussing sexual issues, as well as normative pressures, or a perception, which can be both positive or negative, around what others, including patients and other healthcare professionals might think. Perceived control in relation to the individual healthcare professional's ability to perform the action or behaviour of using the framework in practice is also an important determinant.

\subsection{Description of the final conceptual framework}

Findings from initial development and testing of the preliminary framework were summarised and key components were selected using an iterative approach. This process was led by the research team, with input from members of the expert panel. The panel included patients, healthcare professionals with expertise in prostate cancer care, health communication, patient education and behaviour change techniques and principles. During this phase, multiple versions were drafted, and iteratively reviewed with changes to the content and structure being made at each step. The confirmed sections and components of each section were then combined to generate the final 
version of the EASSi (Engagement, Assessment, Support and Sign-posting) framework (See figure 1).

The overall development process indicated a requirement for a framework to promote engagement and ensure sexual wellbeing support is routinely provided in prostate cancer care. The final conceptual framework intends to achieve this aim by providing a mechanism to ensure sexual wellbeing is consistently raised as a topic for discussion at all stages of care, including at pre-treatment and early post-treatment phases. The EASSi framework also ensures the impact of common treatment-associated sexual concerns is widely acknowledged. In addition, it includes fundamental but individualised support that can be delivered feasibly by all healthcare professionals during brief appointments. The framework can therefore be regarded as a foundation level component of overall sexual wellbeing support and management and a pathway to more intensive ongoing support services where needed. The framework complements existing models as it is designed to assist all clinicians, working in any setting (including those with limited experience or exposure to managing sexual wellbeing issues in practice) to engage with men and partners, discuss sexual issues and provide an 'essential' level of sexual wellbeing support as part of routine practice.

The 'Engagement' section was intended to include an acknowledgement: that sexual wellbeing is not easy to discuss; that sexual side-effects of treatment can have a substantial effect on how men see themselves as individuals; that relationships (both established and casual) can be affected and that sexual issues can lead to reduced or stopped sexual activity. However, a key facet of this section and the overall framework was that the facilitated discussion should also engender a sense of hope and introduce wider sexual thinking beyond focusing on erectile dysfunction. The 'Assessment' section was included primarily to ensure information and support provided during the discussion could be personalised by including information on different side-effects based on treatment type. For example, providing information on bowel sensitivity for patients undergoing radiotherapy. The assessment was also intended to be brief and exploratory only, thereby avoiding issues perceived to more 'sensitive', such as current or previous sexual activity. The 'Support' section was aimed at providing tailored information on common sexual challenges and at normalising sexual concerns. In addition, it was intended to provide an 
acknowledgement of the sense of loss or indeed grief that may be experienced by men and their partners, while also providing information on strategies to cope with this potential loss. A key aim of the support section was to promote resilience and persistence. An important tenant of the section was inclusion of information relevant to the need for those in any current or future relationship to work together and adopt a willingness to try new approaches to manage sexual side-effects of treatment. The final 'Sign-posting' section was aimed at providing more in-depth support, including sources of additional information, online self-management, and erectile dysfunction clinic information. It was intended to provide a mechanism by which the healthcare professional, the patient and the partner could discuss and select appropriate resources based on the content of the sexual wellbeing discussion.

Key changes between the preliminary and final framework and the rationale for the modifications made are detailed below.

\subsection{Modifications to the final framework based on feedback from testing}

Testing provided additional insight into the experiences of using the EASSi framework and sign-posting sheet in practice, with healthcare professionals finding the approach to be valuable in time-constrained clinical settings. Content reviews and 'think-aloud' tests using the framework were carried out including healthcare professionals and patients (see table 1). A key finding from this testing was that patients were more engaged with the frameworks content than healthcare professionals had thought they would be and that use of the framework facilitated improved face-to-face communication. A further observation was that the printed sheet given during the 'Sign-posting' section of the framework was not used routinely.

Key changes based on feedback and testing included reducing the length of the engagement section. The assessment questions were also reduced to include only questions asking about treatment, relationship status and phase of care. Additional questions around ethnicity and sexual orientation were omitted since they were not needed for the type of information and support that the final framework provided. However, to ensure relevant information and support is still provided, online resources were included on the printed sheet given during the 'Sign-posting' section of the framework. Due to the uncertainty around the purpose of the sheet, it was also 
modified by reducing the overall number of resources included on the sheet from nine to seven, and by highlighting a key online self-management programme so that it is provided to all patients, with other resources selected based on patient need and choice. A couple's communication activity was also included on the reverse of the signposting sheet. Detailed information on erectile dysfunction clinics, including their staffing and procedures, was also removed from the 'Support' section due to a lack of routine availability of these services. The overall approach was also amended by using more reflective language. For example, replacing the suggested wording where providing information on side-effects in the support section with 'some men tell us that....' rather than 'what you should know'. Information on the number of side-effects in the support section were also reduced, primarily to reduce potential overloading of information while ensuring discussions are still meaningful.

To support use of the framework, a practical face-to-face training approach was developed based on why each component part of the tool is needed, and on how each part can be used. This training was aimed at supporting healthcare professionals to use the framework and sign-posting sheet. This training also included relevant background information and suggested methods for integration into everyday practice. In addition, a simplified single page version of the framework was developed to provide a brief prompt or reminder that could be used as a poster in clinical areas to remind healthcare professionals to include all four components in sexual wellbeing discussions. The training and healthcare professional perspectives on feasibility and acceptability were evaluated at a 2-hour facilitated workshop attended by 21 clinical nurse specialists which included small group discussions and demonstrations. In addition, attendees completed a modified sexual attitudes and beliefs survey [40] prior to and immediately after the workshop. Analysis of this data using paired sample ttests indicated that the brief training in use of the EASSi framework led to a significant positive change in overall sexual attitude scores, as well as significant improvement in participant's confidence that they would not have any difficulty talking to patients about sexual wellbeing $(t=-2.35 ; P=0.02)$, and an increase in agreement with the statement that discussing sexual concerns with patients is part of their job $(t=-2.16 ; P=0.04)$. Participants reported that the framework had potential to be valuable to their own practice and highlighted the importance of providing greater depth to conversations about sexual concerns. They also indicated how the framework could widen the scope 
of such discussions beyond erectile function. Another common issue raised was that the structured format was useful for providing 'prompts' at each stage of the conversation, facilitating discussions which were seen as sometimes being difficult to initiate and sustain.

\subsection{Linking theoretical domains to the conceptual framework}

The main health professional behaviours which the framework seeks to modify were firstly; to ensure they engage with all men, regardless of phase of care, treatment type, or assumptions about the need to discuss sexual wellbeing with the individual patient. A second behaviour was to ensure all men are provided with some support and advice on potential side-effects. A third behaviour targeted was to ensure that all men are provided with some ongoing support through provision of a sign-posting sheet, pointing towards useful resources including self-management. Finally, a fourth behaviour targeted was to ensure there is a pathway to more in-depth, specialist support services where appropriate. These behaviours were derived from clinical practice recommendations $[8,9]$ and were selected as there is supporting evidence that they could be potentially modifiable and targeted at healthcare professionals working in different prostate cancer care settings. Barriers and enablers impacting on implementation of the target behaviours were identified through analysis of the individual semi-structured interviews and group discussions. These factors were explored based on their underpinning theories and any relevant behaviour change techniques. Details on possible barriers to use of the framework and potential methods to overcome these barriers are shown in table 3. Behaviour change technique selection was determined by feedback and consensus decisions among the research team on likely effectiveness. Potential mechanisms supporting use of the framework included use of a delivery mode able to support use in practice (See figure 2). Key principles and characteristics of the final framework are summarised in Table 4.

Proposed outcomes for assessing pathways mediating change in practice, and for evaluating use of the conceptual framework at the individual level include healthcare professional and patient sexual attitudes and beliefs, self-efficacy for discussing sexual concerns in clinical practice and satisfaction with framework content and structure. System level outcomes include number of patients where the EASSi framework is used and were sexual wellbeing is discussed, change in numbers of 
patients using additional resources or being referred to other services and support, and assessment of the impact of framework use on clinical workload (See figure 2).

\section{Discussion}

This paper describes the systematic development process and the structure and content of a conceptual framework for facilitating sexual wellbeing discussions in routine prostate cancer care. The framework provides a mechanism to improve sexual wellbeing communication through provision of fundamental support delivered routinely, in a manner that still provides tailored advice based on treatment and relationship status. The EASSi conceptual framework, consisting of 'Engagement' [E] 'Assessment' [A], information and 'Support' [S], and 'Sign-posting' [Si] sections, further developed the earlier preliminary framework by refining its content to ensure only core content was included. The rationale for the EASSi framework was based on providing a mechanism to ensure sexual wellbeing is consistently raised as a topic for discussion at all stages of care. The framework can therefore be regarded as a foundation level component of overall, ongoing sexual wellbeing support and management. In this manner, it provides a flexible and responsive approach to deliver standardised but individualised levels of support that can be delivered feasibly by all healthcare professionals during routine appointments. Previous evidence highlights patient dissatisfaction associated with a perception that healthcare professionals are unwilling to discuss sexuality [41]. Use of the EASSi framework is intended to prompt important changes in clinical practice by ensuring essential sexual wellbeing support is provided to all men receiving prostate cancer care, and their partners.

Previous sexual care models [24,25] place an emphasis on healthcare professionals seeking explicit permission or approval to talk about sexual concerns at any point where it might be discussed and consider timing by ensuring patients are 'ready' to discuss sexual concerns. While the framework draws on some aspects of these existing models, it attempts to build upon them by ensuring wider access to routine sexual care and support in prostate cancer care. Its theoretical underpinning may actually be more directly related to brief behaviour change models such as the 5 A's model [42] which has been used as a framework to guide discussions in behavioural counselling interventions for smoking cessation and weight loss. The model stands for 'Ask', 'Advise', 'Assess', 'Assist', and 'Arrange' and is designed to be used briefly in 
around three minutes [43]. Evidence indicates that the framework is most effective when all component parts are completed as part of a single, brief discussion [44].

Preliminary testing of the EASSi framework during development provides initial evidence supporting its use in prostate cancer care settings. The framework may be able to enhance communication around sexual wellbeing and improve support provided by ensuring more routine engagement and delivery of information on potential sexual side effects of treatment, and methods to address these concerns. Ensuring that discussions take place prior to or early during treatment can also be an important part of preparation for alterations to sexual function, managing expectations around recovery and providing a clear rationale for management approaches aimed at erectile dysfunction recovery [45-47]. A particular strength of the EASSi framework approach is the inclusion of a brief engagement and assessment section, alongside a level of support which can be provided based on need. Onward referral to other more specialist services is also included within the framework but other, readily accessible support options are also included. including online self-management resources designed for men and partners. Another strength of the EASSi approach is its flexibility, with scope to facilitate a brief conversation or used as part of a more involved discussion if time allows. The development process itself was also highly systematic and involved multiple user perspectives at all stage in the process, including those of patients and a range of professional groups. Another strength of the development process was use of the theoretical domains approach as a guide [32]. Development of the framework drew on theory, evidence, and exploration of key implementation issues to explore factors that might influence clinical behaviour. Testing of the framework in several stages provided valuable information about the feasibility of using the EASSi framework in practice. Unlike other sexual care models, the framework can be used across settings and without specific training or expertise in sexual care counselling. In addition, it includes evidence-based behavioural change elements. The brevity of the EASSi framework and the combination of a routine assessment alongside provision of appropriate support also means it can be used at any stage in care, from pre-treatment to longer-term follow-up.

One limitation that should be considered is that patients and healthcare professionals involved in development of the framework were predominantly individuals with a strong 
clinical interest in the area of practice. Another potential limitation is that the development process involved patients and healthcare professionals primarily from the UK and the US, potentially limiting the relevance of the framework to other healthcare systems. Work is underway however, to explore the feasibility of using the framework in other contexts and settings. Additional work is also being carried out to test the feasibility of using a modified framework for delivery of other information and support types, including brief lifestyle advice.

To support healthcare professionals, the framework includes resources that can be giving to patients in an effort to reinforce key messages; as well as pointing to effective evidence based self-management resources. Development highlighted a number of important implementation factors, including that healthcare professionals may find it difficult to incorporate the framework, in its current format, into everyday practice. This implementation issue will be explored as part of formative evaluation of a tablet-based EASSi engagement tool tested across different clinical contexts, including in primary care and other cancer care settings. Other issues central to implementation included the individual attitudes and beliefs around sexual wellbeing that some healthcare professionals held. A brief training approach was therefore developed to promote implementation. As part of the framework development process, this training was initially developed and tested as a facilitated face-to-face workshop. However, this delivery mode is not feasible for wider implementation. This training will instead be developed as an e-learning module to ensure wider access for healthcare professionals supporting men with prostate cancer.

\subsection{Conclusion}

Existing sexual care models typically require more intensive intervention and are not ideally suited to use across clinical settings. Moreover, there is evidence that implementation of such models and frameworks in practice is limited. A series of evidence reviews and qualitative data exploring perspectives of key stakeholders highlighted a need for a conceptual framework to promote effective conversations with patients. It was identified that this should be brief, support healthcare professionals to initiate discussions around sexual concerns, address barriers to sexual wellbeing discussions and normalise sexual issues. The initial outline for the framework was based and an assumption that the first step is putting the topic 'on the table' for 
discussion. An additional feature was that the framework should promote a 'shared conversation' between the health professional and patient, and that suitable language, appropriate content and some degree of support should be provided. A need was also identified for the framework to provide additional resources to which men, and partners, could be referred as part of routine sexual wellbeing discussions. Overall findings from the qualitative interviews and other feedback suggested that the final version of the EASSi framework was focused on creating the context to discuss sexual concerns and establish a degree of trust. Furthermore, the importance of active patient involvement in the discussion, as well as involvement of partners was also highlighted. Use of person-centred strategies to engage, assess, support and sign-post were also seen as critical.

\subsection{Practice implications}

The EASSi framework provides a brief, practical format to guide sexual wellbeing discussions in routine clinical practice. It includes four component parts, each designed to be used in sequence. The framework also includes tangible take home messages in the form of a printed handout or sign-posting sheet. The framework is at an appropriate level for use in routine practice and also appears to be acceptable to patients, partners and healthcare professionals. Its use may promote engagement around sexual wellbeing and ensure fundamental but individualised support is provided, potentially addressing current gaps in the routine provision of sexual wellbeing support for men with prostate cancer.

\section{Acknowledgements}

The authors would like to thank the Movember Foundation and Prostate Cancer UK for providing funding and support for the TrueNORTH maximising sexual wellbeing project which this study is part of. The authors would also like to thank all individuals who provided expertise and input during the design and development phase of the project, as well as the healthcare professionals and patient volunteers who participated in interviews and testing.

\section{Competing interests}

The authors declare they have no competing interests. 


\section{Authors' contributions}

EMcC, KP, RM, MS, ST, SJ and MK developed the original proposal for the study and the preliminary version of the framework. EMcC, CF, NB and SOC drafted and revised additional versions of the conceptual framework. EMcC, CF, RM, JC and SOC conducted usability tests and think-aloud interviews. SJ acted as chief investigator at the Northern Ireland Cancer Centre where testing and think-aloud interviews were held. SOC, KP and EMcC, drafted the initial manuscript. All authors revised the manuscript for important intellectual content and approved the final version.

\section{References}

1. KD Miller, L Nogueira, AB Mariotto, et al. Cancer treatment and survivorship statistics, 2019. CA Cancer J Clin. (2019) Jun 11 [Epub ahead of print]

2. F Pishgar, H Ebrahimi, S Saeedi Moghaddam, C, et al. Global, Regional and National Burden of Prostate Cancer, 1990 to 2015: Results from the Global Burden of Disease Study 2015. J Urol. 199 (2018) 1224-1232.

3. JL Donovan, FC Hamdy, JA Lane, et al. Patient-Reported Outcomes after Monitoring, Surgery, or Radiotherapy for Prostate Cancer. N Engl J Med. 13 (2016) 1425-1437.

4. A Downing, P Wright, L Hounsome, et al. Quality of life in men living with advanced and localised prostate cancer in the UK: a population-based study. Lancet Oncol. 20 (2019) 436-447.

5. MK Haahr, NH Azawi, LG Andersen, et al. A Retrospective Study of Erectile Function and Use of Erectile Aids in Prostate Cancer Patients After Radical Prostatectomy in Denmark. Sex Med. 5 (2017) e156-e162.

6. TW Gaither, MA Awad, EC Osterberg, et al. The Natural History of Erectile Dysfunction After Prostatic Radiotherapy: A Systematic Review and MetaAnalysis. J Sex Med.14 (2017) 1071-1078.

7. M Brundage, MR Sydes, WR Parulekar, et al. Impact of Radiotherapy When Added to Androgen-Deprivation Therapy for Locally Advanced Prostate Cancer: Long-Term Quality-of-Life Outcomes From the NCIC CTG PR3/MRC PR07 Randomized Trial. J Clin Oncol. 33 (2015) 2151-2157.

8. National Institute for Health and Care Excellence. Prostate cancer: diagnosis and management. Clinical guideline [NG131]. May

2019. https://www.nice.org.uk/guidance/ng131. Accessed: May 2019. 
9. MJ Resnick, C Lacchetti, DF Penson; American Society of Clinical Oncology. Prostate cancer survivorship care guidelines: American Society of Clinical Oncology practice guideline endorsement. J Oncol Pract. 11 (2015) e445-449.

10. NJ Sporn, KB Smith, WF Pirl, et al. Sexual health communication between cancer survivors and providers: how frequently does it occur and which providers are preferred? Psychooncology. 24 (2015) 1167-73.

11. KE Flynn, JB Reese, DD Jeffery DD, et al. Patient experiences with communication about sex during and after treatment for cancer. Psychooncology 21 (2012) 594-601

12. A Mehta, CE Pollack, TW Gillespie et al. What Patients and Partners Want in Interventions That Support Sexual Recovery After Prostate Cancer Treatment: An Exploratory Convergent Mixed Methods Study. Sex Med. 2019;7(2):184-191.

13. JO Julien, B Thom, NE Kline. Identification of barriers to sexual health assessment in oncology nursing practice. Oncol Nurs Forum. 37 (2010) E186-190.

14. P Singy, C Bourquin, B Sulstarova, et al. The impact of communication skills training in oncology: a linguistic analysis. J Cancer Educ. 3 (2012) 404-408.

15. I Strada, E Vegni, G Lamiani. Talking with patients about sex: results of an interprofessional simulation-based training for clinicians. Intern Emerg Med. 11 (2016) 859-866.

16. G Harrison, S Hayden, V Cook. A Cushing. Improving health care professionals' feedback on communication skills: development of an on-line resource. Patient Educ Couns. 88 (2012) 414-9.

17. L Forbat, I White, S Marshall-Lucette, et al. Discussing the sexual consequences of treatment in radiotherapy and urology consultations with couples affected by prostate cancer. British Journal of Urology International. 109 (2012) 98-103.

18. PM Moore, S Rivera Mercado, M Grez Artigues, et al. Communication skills training for healthcare professionals working with people who have cancer. Cochrane Database Syst Rev. 28 (2013)

19. ES Zhou, L Nekhlyudov, SL Bober. The primary health care physician and the cancer patient: tips and strategies for managing sexual health. Transl Androl Urol. 4 (2015) 218-231.

20. LV van de Poll-Franse, KA Nicolaije, NP Ezendam. The impact of cancer survivorship care plans on patient and health care provider outcomes: a current perspective. Acta Oncol. 56 (2017) 134-138.

21. SA Lenzen, R Daniëls, MA van Bokhoven MA, et al.Development of a conversation approach for practice nurses aimed at making shared decisions on 
goals and action plans with primary care patients. BMC Health Serv Res. 18 (2018) 891.

22. RD Vromans, MC van Eenbergen, SC Pauws, et al. Communicative aspects of decision aids for localized prostate cancer treatment - A systematic review. Urol Oncol. 37 (2019) 409-429.

23. JS Annon. PLISSIT therapy. In: Corsine RJ, editor. Handbook of Innovative Psychotherapies. New York: Wiley and Sons; 1981. p. 629-39.

24. B Taylor, S Davis. Using the extended PLISSIT model to address sexual healthcare needs.

Nurs Stand. 21 (2006) 35-40.

25. J Mick, M Hughes, MZ Cohen. Using the BETTER Model to assess sexuality. Clin J Oncol Nurs. 8 (2004) 84-86.

26. LM Walker, E Wiebe, J Turner, et al. The Oncology and Sexuality, Intimacy, and Survivorship Program Model: An Integrated, Multidisciplinary Model of Sexual Health Care within Oncology. J Cancer Educ. (2019) Dec 3. [Epub ahead of print]

27. K Parahoo, S McDonough, E McCaughan, et al. Psychosocial interventions for men with prostate cancer: a Cochrane systematic review. BJU Int. 116 (2015) 174183.

28. E McCaughan, S McKenna, O McSorley et al. The experience and perceptions of men with prostate cancer and their partners of the CONNECT psychosocial intervention: a qualitative exploration. J Adv Nurs. 71 (2015) 1871-1882.

29. K Parahoo, S McKenna, G Prue et al. Facilitators' delivery of a psychosocial intervention in a controlled trial for men with prostate cancer and their partners: a process evaluation. J Adv Nurs. 73 (2017) 1620-1631.

30. TA Skolarus, JA Ragnoni, C Garlinghouse, et al. Multilingual Self-Management Resources for Prostate Cancer Survivors and Their Partners: Results of a LongTerm Academic-State Health Department Partnership to Promote Survivorship Care. Urology. 110. (2017) 92-97.

31. Wittmann D. Emotional and sexual health in cancer: partner and relationship issues. Curr Opin Support Palliat Care. 10 (2016) 75-80.

32. SD French, SE Green, DA O'Connor, et al. Developing theory-informed behaviour change interventions to implement evidence into practice: a systematic approach using the Theoretical Domains Framework. Implement Sci. 24 (2012) 38.

33. SR O'Connor, J Connaghan, R Maguire, et al. Healthcare professional perceived barriers and facilitators to discussing sexual wellbeing with patients after diagnosis of chronic illness: A mixed-methods evidence synthesis. Patient Educ Couns. 102 (2019) 850-863. 
34. P Sheeran, A Maki, E Montanaro et al. The impact of changing attitudes, norms, and self-efficacy on health-related intentions and behavior: A meta-analysis. Health Psychol. 35 (2016) 1178-1188.

35. M Conner, $R$ McEachan, $R$ Lawton, $P$ Gardner. Basis of intentions as a moderator of the intention-health behavior relationship. Health Psychol. 35 (2016) 219-227.

36. S Houlihan. Dual-process models of health-related behaviour and cognition: a review of theory. Public Health. 156 (2018) 52-59.

37. M Hennessy, A Bleakley, M Ellithorpe. Prototypes reflect normative perceptions: implications for the development of reasoned action theory. Psychol Health Med. 23 (2018) 245-258.

38. R Khanna, J Kavookjian, VG Scott et al. Using the theory of reasoned action to determine physicians' intention to measure body mass index in children and adolescents. Res Social Adm Pharm. 5 (2009) 170-181.

39. L Ross, CL Kohler, DM Grimley, et al The theory of reasoned action and intention to seek cancer information. Am J Health Behav. 31 (2007) 123-134.

40. KE Reynolds, MA Magnan. Nursing attitudes and beliefs toward human sexuality: collaborative research promoting evidence-based practice. Clin Nurse Spec. 19 (2005) 255-259.

41. E Gilbert, J Perz, JM Ussher. Talking about sex with health professionals: the experience of people with cancer and their partners. Eur J Cancer Care (Engl). 25 (2016) 280-293.

42. EP Whitlock, CT Orleans, N Pender, et al. Evaluating primary care behavioral counseling interventions: an evidence-based approach. Am J Prev Med. 22 (2002) 267-284.

43. J Asselin, E Salami, AM Osunlana, et al. Impact of the 5As Team study on clinical practice in primary care obesity management: a qualitative study. CMAJ Open. 26 (2017) E322-E329.

44. RE Glasgow, S Emont, DC Miller. Assessing delivery of the five 'As' for patientcentered counseling. Health Promot Int. 21 (2006) 245-255.

45. ID White, J Wilson, P Aslet, et al. Development of UK guidance on the management of erectile dysfunction resulting from radical radiotherapy and androgen deprivation therapy for prostate cancer.

Int J Clin Pract. 69 (2015) 106-123.

46. MG Kirby, ID White, J Butcher et al. Development of UK recommendations on treatment for post-surgical erectile dysfunction. Int J Clin Pract. 68 (2014) 590-608. 
47. AER Faris, DK Montague, BC Gill. Perioperative Educational Interventions and Contemporary Sexual Function Outcomes of Radical Prostatectomy. Sex Med Rev. 7 (2019) 293-305. 
Table 1. Qualitative methods used during development of the conceptual framework

\begin{tabular}{|c|c|c|}
\hline Qualitative method & Participants (N) & Numbers \\
\hline $\begin{array}{l}\text { Interactive stakeholder worksh } \\
\text { with group discussion* }\end{array}$ & $\begin{array}{ll}\text { oTo identify requirements and expected components of thePatients }(5) \\
\text { proposed framework } & \text { Partners (3) } \\
& \text { Healthcare professionals }{ }^{* *}(16)\end{array}$ & 24 \\
\hline $\begin{array}{l}\text { Individual semi-structured } \\
\text { interviews }\end{array}$ & $\begin{array}{l}\text { To assess views on current practice, and explore barriersPatients (5) } \\
\begin{array}{ll}\text { and facilitators to sexual wellbeing discussions inPartners (1) } \\
\text { practice } & \text { Healthcare professionals }{ }^{* *}(5)\end{array}\end{array}$ & 11 \\
\hline Group discussion & $\begin{array}{l}\text { To explore barriers and facilitators to sexual wellbeingHealthcare professionals** }(5) \\
\text { discussions in practice }\end{array}$ & 5 \\
\hline Think-aloud interviews & 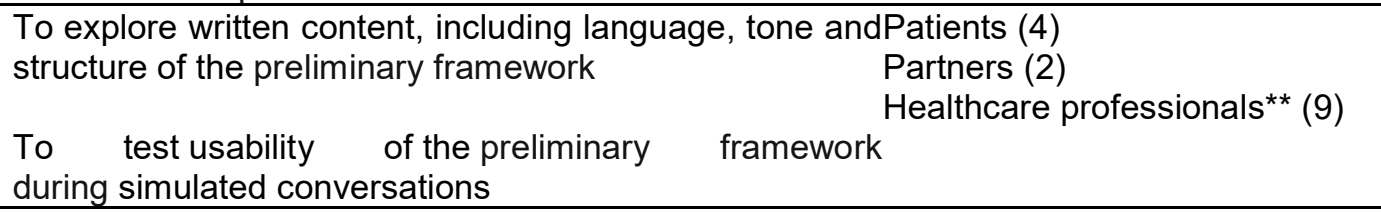 & 15 \\
\hline $\begin{array}{l}\text { Training workshop with small } \\
\text { group discussions }\end{array}$ & $\begin{array}{l}\text { To explore barriers and facilitators to implementation ofClinical nurse specialists (21) } \\
\text { the framework in practice and assess impact of training } \\
\text { on sexual attitudes and beliefs }\end{array}$ & 21 \\
\hline
\end{tabular}

* Stakeholders included men with lived experience of prostate cancer, partners, Healthcare professionals and policy makers.

** Healthcare professionals included doctors, nurses, allied health professionals.

$\mathrm{N}$ : Number 
Table 2. Aims and suggested components of the preliminary conceptual framework for improving routine sexual wellbeing communication in prostate cancer care

\author{
Key aims of the framework \\ To provide a professional, secure \\ approach to initiating sexual wellbeing \\ discussions \\ To prepare health professionals for the \\ conversation \\ To ensure (using a structured process) that \\ all men (and partners) receive some sexual \\ wellbeing support \\ To ensure the patient is aware sexual \\ wellbeing is going to be discussed
}

To provide an early opportunity for issues to be raised by the patient or partner

An assessment should identify:

Any treatment received

Phase of care

Sexual orientation

Ethnic background

Relationship status

\section{Suggested components to achieve aim}

Include language normalising sexual concerns as a symptom that needs addressing

- Use language that quickly puts the topic 'on the table'

- Inform the patient that sexual concerns are open for discussion

- Include points where men can ask questions

- Provide basic information on opening conversation at a future time or on 'how to have a conversation with a healthcare professional'

- Include acknowledgment of the importance of sexual wellbeing and the sensitivity of the topic

- Include recognition of partner role

- Reassure on frequency of sexual concerns after diagnosis

- Provide a holistic assessment and approach by including questions on treatment type and psychosocial factors including sexual orientation, and relationship status

\section{Source of supporting evidence}

Qualitative interviews

Qualitative interviews

Qualitative interviews

Evidence reviews
Evidence reviews

Evidence reviews

Stakeholder workshop

Qualitative interviews

Evidence reviews

Evidence reviews

Stakeholder workshop

Qualitative interviews 
To provide a 'guideline' for giving sexual

To provide reassurance that concerns are normal after diagnosis and treatment wellbeing support

- Provide a comprehensive assessment to guide personalized support and sign-posting

- Normalise the assessment so it is treated like other side-effects of treatment

- Quickly provide standardised, essential information

- $\quad$ Provide a mechanism to identify (limited, relevant) other concerns or current side effects of treatment

- Identify any associated distress / depression / anxiety / unrealistic expectations

- Identify importance of sexual concerns to patient

- $\quad$ Identify needs of patient and partner

- Identify possible barriers to engagement

To provide personalized support to men and partner

To provide detail on what the long-term support options will be

To manage patient expectations about recovery

To emphasis the ongoing sexual recovery 'journey'

To introduce that possibly of a wider concept of sex and sexuality
- Provide information on how sex life will be changed

- Include detail on sexual recovery as an ongoing journey

- Provide detail on expectations and potential side-effects of treatment

- $\quad$ Provide ongoing reminders or prompts

- $\quad$ Provide resources that the partner can use if not present at appointment
Evidence reviews

Qualitative interviews

Evidence reviews

Qualitative interviews

Stakeholder workshop

Stakeholder workshop

Qualitative interviews

Qualitative interviews

Evidence reviews

Evidence reviews

Stakeholder workshop

Evidence reviews

Qualitative interviews

Evidence reviews

Qualitative interviews

\section{Evidence reviews}

Qualitative interviews

Evidence reviews 
To provide access to further support-based on need
- Point to other to appropriate resources which may be useful at other phases of care

- Promote ongoing support available based on need
Qualitative interviews

Evidence reviews

Qualitative interviews 
Table 3. Components of the conceptual framework included to address barriers and facilitators influencing implementation

\section{Identified barrier or facilitator to use ofRelevant Theoretical Domain [28]}

\section{the framework}

Limited awareness of sexual wellbeing1. Knowledge

concerns and the impact on patients

Beliefs about negative consequences of6. Beliefs about Consequences

raising sexual wellbeing as a topic

Stage of care can be variable and patient11. Environmental context and resources

information needs may differ

(including patients in a pre-treatment

phase)

Limited time to address sexual concerns11. Environmental context and resources

during appointments

Perception that patients will not wish to6. Beliefs about Consequences

discuss sexual wellbeing due to sensitivity12. Social influences

of topic

Perception that discussing sexual11. Environmental context and resources

wellbeing with patients at early stage of

treatment or soon after diagnosis is not a

priority

Perception that providing sexual3. Social/professional role and identity

wellbeing support is a specialist role (or

role of other healthcare profession)

Perception that addressing sexual3. Social/professional role and identity wellbeing will affect how colleagues view6. Beliefs about Consequences

them professionally

Detailed assessment is needed to6. Beliefs about Consequences

evaluate patient needs

11. Environmental context and resources

1. Knowledge

Assessment can introduce concerns that6. Beliefs about Consequences

health professionals do not have time or11. Environmental context and resources

resources to address
Intervention components including behaviour change techniques*

Information about consequences (5.1) through use of framework and healthcare professional training

Information about consequences (5.1 and 5.3) through healthcare professional training and behavioura experiments (4.1) by observing patient responses during use of framework

Instruction on how to perform the behaviour (4.1) and demonstration / modelling of behaviour (6.1) through use of framework which provides information based on patient phase of care

Information about consequences (5.1) and prompts and cues (7.1) using patient hand-out taken from appointment and use of framework

Information about consequences (5.1) through use of framework and healthcare professional training

Information about consequences (5.1) through use of framework and healthcare professional training

Social comparison (6.1) though healthcare professional training

Social comparison (6.1) though healthcare professional training and restructuring environment (12.1) through use of framework in practice

Instruction on how to perform the behaviour (4.1) and demonstration / modelling of behaviour (6.1) through use of framework

Instruction on how to perform the behaviour (4.1) and demonstration / modelling of behaviour (6.1) through use of framework 
Skills and beliefs about ability to provide4. Beliefs about capabilities

appropriate or effective sexual care and11. Environmental context and resources

support

2. Skills

Patients may only need additional11. Environmental context and resources

resources at a later point in care

Patients not remembering information, or1. Knowledge

which additional support resources are10. Memory, attention and decision

recommended

\section{processes}

11. Environmental context and resources
Instruction on how to perform the behaviour (4.1) and demonstration / modelling of behaviour (6.1) through use of framework

Prompts and cues (7.1) using patient hand-out taken from appointment

Prompts and cues (7.1) using patient hand-out taken from appointment

* Michie Set al. The behavior change technique taxonomy (v1) of 93 hierarchically clustered techniques: building an international consensus for the reporting of behavior change interventions. Ann Behav Med. 2013 Aug;46(1):81-95. 
Table 4. Key principles and characteristics of the final version of the EASSi conceptual framework

\section{Objectives of EASSi framework}

To promote routine engagement and ensure men with prostate cancer have a 'meaningful' conversation abound sexual wellbeing after diagnosis (so all patients have access to essential information and support)

To provide a structure to sexual wellbeing conversations that includes a brief, standardised means of opening the conversation (putting the topic on the table)

To ensure a minimal level of psychosexual support is provided (which is relevant at all phases of care)

To ensure routine sign-posting to appropriate resources (providing additional support, where needed and option to recap information discussed during the appointment)

\section{Key features of EASSi framework included to meet objective}

- Uses a simple format which can be used easily as part of routine appointments

- $\quad$ Can be used to 'pre-plan' sexual wellbeing conversations

- Can be used to 'restructure' the clinical setting or environment by providing a 'shared facility' which ensures the patient and healthcare professional take part in a joint discussion

- Use of the framework ensures time is given to discussing sexual wellbeing during brief routine appointments

- Provides appropriate terms relevant to opening sexual wellbeing conversations

- Provides 'tailoring' of information and support based on need

- Provides 'layering' of information based on need (ensuring framework can be brief but can facilitate a longer conversation if indicated)

- Provides referral to appropriate services based on need

- Provides access to a more 'in-depth' online self-management programme to provide longer-term support

- Includes printed handout for patients to take away from appointment as a reminder of key points 
Figure 1. Purpose and outline content of the EASSi framework components

\begin{tabular}{|c|c|c|}
\hline Section & Rationale for section and purpose & Outline of section content \\
\hline Engagement & \multirow{2}{*}{$\begin{array}{l}\text { Sexual dysfunction is a major problem } \\
\text { and is often not addressed } \\
\text { Section used to: } \\
\text { Normalise sexual concerns } \\
\text { Explain that a brief conversation about } \\
\text { sex and prostate cancer will take place }\end{array}$} & \multirow{2}{*}{$\begin{array}{l}\text { Includes explanation that: } \\
\text { There are many side-effects of prostate cancer treatment } \\
\text { At the end a sheet will be provided with more detailed information and resources } \\
\text { Sex can be a difficult subject which is not easy to talk about, but it is an important } \\
\text { part of life }\end{array}$} \\
\hline & & \\
\hline Assessment & \multirow{2}{*}{$\begin{array}{l}\text { Basic assessment is needed to provide } \\
\text { tailored support } \\
\text { Section used to: } \\
\text { Ask about treatment type } \\
\text { Stage of treatment } \\
\text { Relationship status }\end{array}$} & \multirow{2}{*}{$\begin{array}{l}\text { Section asks three questions including following example: } \\
\text { Have you had any of the following treatments? } \\
\text { 1. Radiotherapy } \\
\text { 2. Hormone therapy } \\
\text { 3. Surgery } \\
\text { 4. Combined radio and hormone therapy }\end{array}$} \\
\hline & & \\
\hline \multirow[t]{2}{*}{ Support } & \multirow[b]{2}{*}{$\begin{array}{l}\text { There are many sexual side-effects of } \\
\text { treatment but also things that can help } \\
\text { Section used to: } \\
\text { Provide information on expected } \\
\text { sexual challenges } \\
\text { Acknowledge sex life will change } \\
\text { Give brief advice on... } \\
\text { What can be done } \\
\text { Widening understanding of sex }\end{array}$} & \multirow[b]{2}{*}{$\begin{array}{l}\text { Section provides advice (based on treatment) including: } \\
\text { List of possible side-effects and some ideas to help. } \\
\text { For example: Hormone side-effect 1: Less interest in having sex } \\
\text { Advice: } \\
\text { The treatment affects the level of testosterone in your body } \\
\text { This has an effect on your sex drive } \\
\text { Even if you have less sex drive, you can still feel pleasure } \\
\text { And gives other advice on: } \\
\text { need to think about sex in new ways, try new things and have patience and } \\
\text { persistence }\end{array}$} \\
\hline & & \\
\hline Sign-posting & $\begin{array}{l}\text { After providing essential support need } \\
\text { to signpost to other services } \\
\text { Section used to: } \\
\text { Point towards additional resources or } \\
\text { services }\end{array}$ & $\begin{array}{l}\text { Section includes provision of printed sign-posting sheet including: } \\
\text { Online self-management } \\
\text { Other information and support resources } \\
\text { Useful tips and exercises } \\
\text { Localised information on erectile dysfunction clinic }\end{array}$ \\
\hline
\end{tabular}


Figure 2. Conceptual framework to improve sexual wellbeing communication in routine prostate cancer care using EASSi

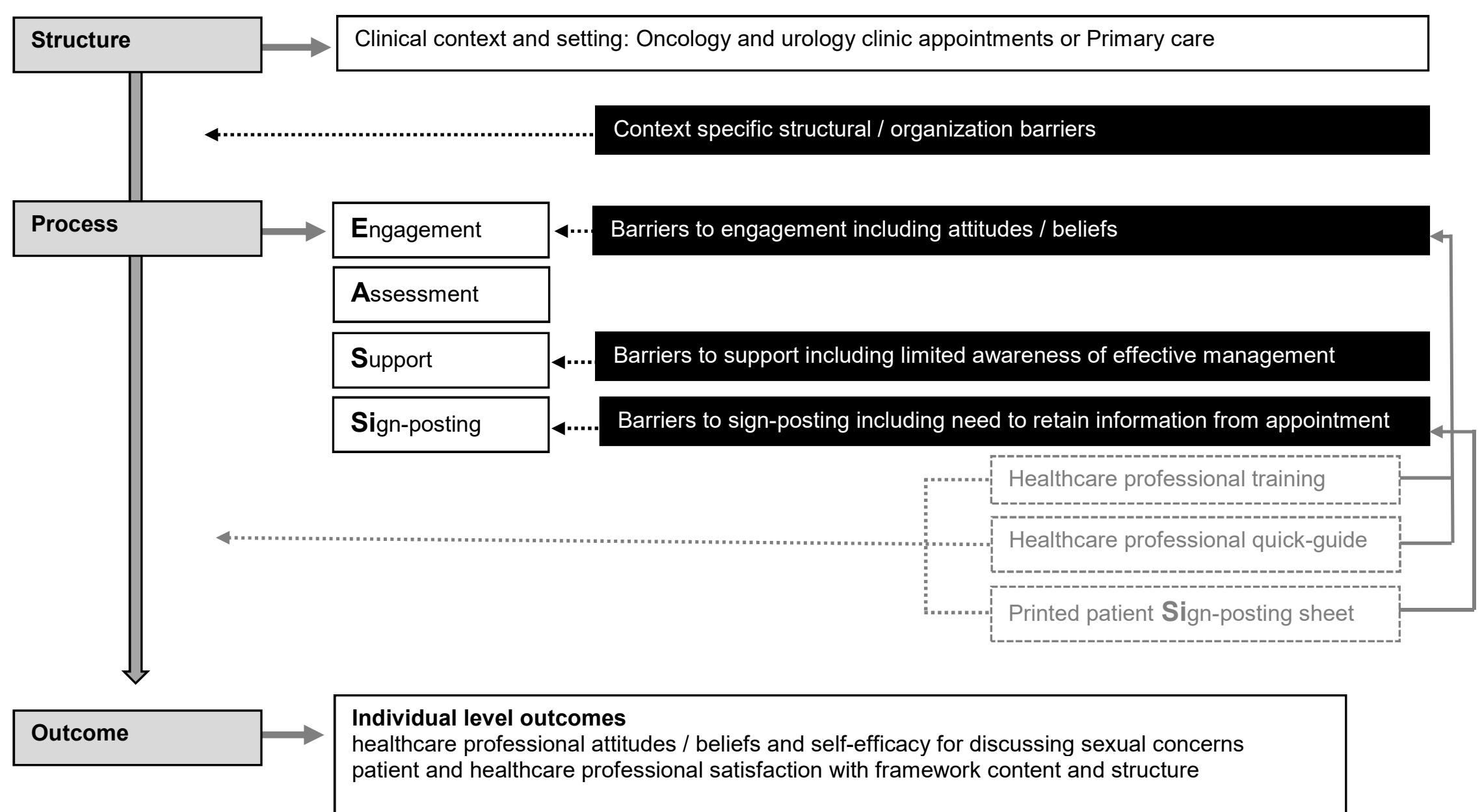

System level outcomes

number of patients receiving foundation level of sexual wellbeing care and support

number of patients using additional resources or referred to other services

Impact of framework usage on clinical workload 


\section{Supplementary file 1: Topic guide for semi-structured interviews}

\section{Questions}

1. Can you tell me about any experience you have of using a 'discussion tool' or conversation aid?

2. What would your expectations of such a tool be?

3. Are these expectations met by the proposed EASSi framework?

4. What parts of the EASSi framework did you like?

5. What would you want to change about the EASSi framework?

6. Is there anything else you would like the EASSi framework to include?

7. What do you think about the activities included in the EASSi framework?

8. What would help people to remember what was discussed after using the framework?

9. Who do you think is best placed to use the EASSi framework?

10. What do you think is the best setting for the EASSi framework to be used?

11. What do you think is the best format for the EASSi framework?

12. What do you think about how long the conversation guided by the framework should be?

13. Is there anything else you'd like to say about the EASSi framework?

14. Do you have any questions? 